\title{
Attainable-Set Model Predictive Control for AUV Formation Control
}

\author{
Rui Gomes \\ Electrical engineering department \\ SYSTEC, Faculty of Engineering, Porto University \\ Porto, Portugal, (rgomes@fe.up.pt)
}

\author{
Fernando Lobo Pereira \\ Electrical engineering department \\ SYSTEC, Faculty of Engineering, Porto University \\ Porto, Portugal, (flp@fe.up.pt)
}

\begin{abstract}
In this article, we focus on the motion control of an AUV formation in order to track a given path along which data will be gathered. A computationally efficient architecture enables the conciliation of onboard resources optimization with state feedback control - to deal with the typical a priori high uncertainty - while managing the formation with a low computational and power budgets. To meet these very strict requirements, a novel Model Predictive Control (MPC) scheme is used. The key idea is to pre-compute data which is known to be time invariant for a number of likely scenarios and store it on-board in appropriate look-up tables. Then, as the mission proceeds, sampled motion sensor data, and communicated data is processed in each one of the AUVs and fed to the onboard proposed MPC scheme implemented with the dynamics of the formation that, by combining with information extracted from the pertinent on-board look-up tables, determine the best control action with inexpensive computational operations.
\end{abstract}

Index Terms-Model predictive control, Attainable set, AUV formation control, Obstacle collision avoidance

\section{INTRODUCTION}

This article concerns the design of a general control scheme satisfying very hard requirements usually arising in networked AUV systems applications such as vehicle formations which are important for data gathering missions. Typically, these requirements consist in the conciliation of the optimization of scarce onboard resources with motion control robustness and adaptivity, while subject to strict real-time constraints due to limited onboard computational resources and power. Figure 1 shows current typical state-of-the-art vehicles, where it is clear that the scarcity of power follows from the need to pack all sub-systems for data gathering and motion autonomy for reasonably long endurance missions in a very small volume.

In order to ensure the above mentioned requirements, a new Model Predictive Control (MPC) scheme - denoted by ASMPC - that, by assuming the reasonable hypothesis of timeinvariance of the dynamics as well as of some key features of the environment, replaces a significant amount of the online computational burden of the conventional MPC, [1]-[3], by off-line computation, being the generated data stored in

The authors acknowledge the partial support of FCT R\&D Unit SYSTEC - POCI-01-0145-FEDER-006933/SYSTEC funded by ERDF - COMPETE2020 - FCT/MEC - PT2020 - extension to 2018, Project STRIDE - NORTE-01-0145-FEDER-000033, funded by ERDF - NORTE 2020, and project MAGIC - POCI-01-0145- FEDER-032485 - funded by FEDER via COMPETE 2020 - POCI, and by FCT/MCTES via PIDDAC.

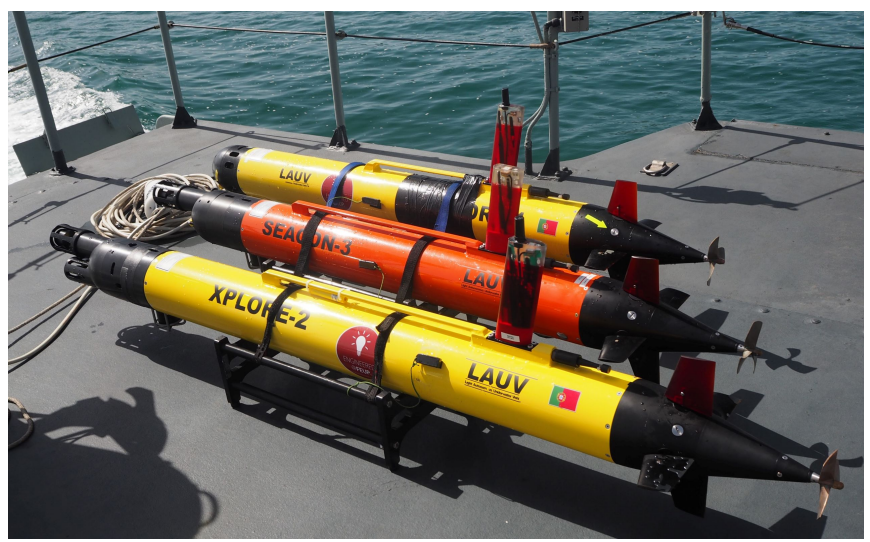

Fig. 1. Set of AUVs (courtesy of LSTS, https://lsts.fe.up.pt)

appropriate onboard look-up tables. In the course of the mission execution, pertinent data from these look-up tables will be retrieved and processed according to the prevailing context perceived by the communicated and sensed data. The AS-MPC is designed in such a way that the necessary on-line processing involves a computational burden significantly lower than the one required by the usual MPC schemes. Properties of closed loop stability, asymptotic optimality, and robustness have been proved, thus showing that the proposed arrangement is able to handle an increasing range of applications for which severe real-time constraints impose on-line computational parsimony in computing state and environment feedback control strategies optimizing the consumption of scarce resources. Moreover, examples illustrating how the AS-MPC scheme accommodates controlled systems with hybrid dynamics that naturally arises in the considered class of applications is presented.

Here, we present further refinements of the control architecture presented in [4] for the management and control of the formation of AUVs. This development is in the sequel of [5] in which an MPC scheme with very low on-line computational budget obtained by taking advantage of time invariance of the dynamics and some environmental features. This allows to pre-compute a priori objects which are key for the on-line feedback control synthesis. This is the essential feature of the AS-MPC developments and are an integral part of the $\mathrm{PhD}$ thesis [6]. Clearly, this approach is substantially distinct from all previous MPC schemes as it is clear from state-of-the-art 
overview in the next section.

In the next section, a typical AUV formation control problem to track a reference trajectory along which payload data is to be gathered, followed by comments on pertinent previous work. The presentation and derivation of the ASMPC scheme for AUV motion control is given in section III. This section also includes an outline of the stability, suboptimality and robustness properties. Then, section IV shows how the proposed framework may accommodate controlled hybrid systems, thus defining a control architecture enabling multiple event-triggered modes of operation. Section V states brief conclusions and comments on future work.

\section{The AUV Formation Tracking Problem}

The formulation of AUV formation control problem can be either centralized or decentralized, depending on whether the data available to each AUV is either global or generated by its neighbours. The overall goal is to control the pattern or patterns of AUVs positions while tracking or following a given reference trajectory or path along which payload data is gathered according to given requirements while accommodating unforseen events such as emergence of obstacles, AUV failures, etc. Below, we state the basic abstract optimal control problem.

$$
\begin{aligned}
\left(P_{T}\right) \text { Minimize } & g_{0}\left(x\left(t_{0}+T\right)\right)+\int_{t_{0}}^{t_{0}+T} g(t, x(t), z(t), \bar{u}(t)) d t \\
\text { subject to } & \dot{x}(t)=f(t, x(t), z(t), \bar{u}(t)) \quad \mathcal{L}-\text { a.e. } \\
& \bar{u}(t) \in \Omega, \quad h(t, x(t)) \leq \quad \mathcal{L}-a . e . \\
& x\left(t_{0}\right)=x_{0}, \quad x\left(t_{0}+T\right) \in C_{t_{0}+T} \leq 0
\end{aligned}
$$

where $g_{0}$ is the endpoint cost functional, $g$ is the running cost integrand, $f, h$, are the vehicle dynamics, and the inequality state constraints functions, $t_{0}$, and $x_{0}$ are the current time and state values, $C_{t_{0}+T}$ is the desired target set at $t_{0}+T$, and $z$ is the reference trajectory to be tracked.

To instance $\left(P_{T}\right)$ in the context of AUV formation of $N$ vehicles tracking a reference trajectory $z$, consider, for the AUV $i$ :

- $x=\operatorname{col}\left(\eta^{i}, \nu^{i}\right)$, and $\bar{u}=\operatorname{col}\left(\tau^{i}\right), i=1, \ldots, N$.

- $g_{0}(\cdot)=0, g(t, x(t), u(t))=\left(\eta^{i}(t)-\eta_{r}^{i}(t)\right)^{T} Q\left(\eta^{i}(t)-\right.$ $\left.\eta_{r}^{i}(t)\right)+\tau^{i T}(t) R \tau^{i}(t)$, where $\eta_{r}^{i}(\cdot)$ is the reference trajectory for the $i^{t h}$ vehicle.

- By considering, for each vehicle (we drop the index $i$ ), the state and the controls given by $\eta=[x, y, \psi]^{T}$ and $\nu=$ $[u, v, r]^{T}$ and $\tau=\left[\tau_{u}, \tau_{r}\right]^{T}$, respectively, the dynamics are given in [7]. For details, [4].

- Other constraint types include: (i) endpoint state constraints, $\eta^{i}(t+T) \in C_{t+T}$, (ii) control constraints, $\tau^{i}(s) \in \mathcal{U}^{i}$, (iii) state constraints, $\left(\eta^{i}(s), \nu^{i}(s)\right) \in \mathcal{S}^{i}$, (iv) communication constraints $g_{i, j}^{c}\left(\eta^{i}(s), \eta^{j}(s)\right) \in C_{i, j}^{c}, \forall j \in$ $\mathcal{G}^{c}(i)$, and $(\mathrm{v})$ formation constraints $g_{i, j}^{f}\left(\eta^{i}(s), \eta^{j}(s)\right) \in C_{i, j}^{f}, \forall j \in \mathcal{G}^{f}(i)$.

The interpretation of the data of this problem has been made in the context of formation control. Each AUV communicates acoustically with its neighbors or all other AUVs according to the communication graph connectivity. Each vehicle is a node of this graph whose arcs are the communication links. The vehicles navigate sufficiently close to each other so that there is no loss of packets. Modes of operation include data gathering, obstacle collision avoidance, communication, and loitering. Each mode of operation requires its own formation pattern.

In the decentralized control version, the feedback control scheme requires two components, one underlying its own motion and that of its neighbors, and another concerning the generation of a consensus to promote the cohesion and evolution of the formation. The information distribution pattern shapes the components of the state and trajectory reference variables.

MPC schemes generate a feedback control synthesis conciliating sub-optimization with discrete feedback control. By and large, the basic versions consists in computing the control action for the current control horizon starting at each sampling time, by solving the on-line optimal control problem $\left(P_{T}\right)$ over the prediction horizon $\left[t_{0}, t_{0}+T\right]$, being the state variable initialized at the current best estimate updated with the latest state sample, $x_{0}$. Then, the obtained optimal control strategy is applied during the first control horizon, $\left[t_{0}, t_{0}+\Delta\right]$, where $\Delta$ is much smaller than $T$. The cycle is repeated after the needed parameters update, [6].

Extended versions of models and control systems of AUVs see [7] for a comprehensive overview - for very diverse robot craft have been considered for single and multiple vehicles. Non-linear control theory and geometric control provide tools that led to very popular design techniques, [8]-[10]. The need of resources optimization and control problem versatility made MPC schemes very popular in this area, [1], [2], [11]-[19]. In this sample of references and work cited therein, issues such as underwater communications failures and delays in continuum and discrete times, centralized and decentralized schemes, linear and nonlinear dynamics, leader-follower and leaderless schemes, collision-free motion, output feedback, cooperative motion, and competitive strategies, single and multiple objectives, as well as a varied range of applications (surveillance, exploration, tracking paths and trajectories), have been considered. However, the typically required intense on-line computational burden seems to have been overlooked and stimulated the emergence of the approach considered in this article.

\section{THE AS-MPC SCHEME}

This section starts with the work described [4], [5] and encompasses with additional properties proved since then. The main idea of the approach consists in solving a sequence of finite dimensional optimization problems instead $\left(P_{T}\right)$ of the usual MPC schemes. This is enabled by the time-invariance data or predictable time evolution of much of the problem data, such as vehicle dynamics, and features of the environment, since it allows the to pre-computation of the attainable set and the value functions associated with $\left(P_{T}\right)$, which are, then, 
stored on-board the AUV to be used for the on-line control synthesis with low cost computational effort.

Let us simplify the notation by omitting the reference trajectory and consider the control variable $u$ instead of $\bar{u}$. Define the Value Function $V_{T}\left(t_{0}, y\right)$ as

$$
\min _{u \in \mathcal{U}, \xi \in C_{t_{0}+T}}\left\{g_{0}(\xi)+\int_{t_{0}}^{t_{0}+T} g(\tau, x(\tau), u(\tau)) d \tau\right\}
$$

subject to $x\left(t_{0}+T\right)=\xi, x\left(t_{0}\right)=y, \dot{x}=f(t, x, u), \mathcal{L}$-a.e., and the Forward Attainable Set by

$\mathcal{A}_{f}\left(t_{0}+\Delta ; t_{0}, x_{0}\right)=\left\{x\left(t_{0}+\Delta\right): \dot{x}=f(t, x, u), u \in \Omega, x\left(t_{0}\right)=x_{0}\right\}$

Let $\Delta<T$. By taking into account the Principle of Optimality (we assume the problem to be positional, [20]), and by performing a straightforward change of state variable, see [4], and by not relabelling, it is easy to conclude that $\left(P_{T}\right)$ is, for the control horizon $\left[t_{0}, t_{0}+\Delta\right]$, equivalent to

$$
\begin{aligned}
\left(P_{T}^{\Delta}\right) \text { Minimize } & \left.V_{T}\left(t_{0}+\Delta, \bar{x}\right)\right) \\
\text { subject to } & \bar{x} \in \mathcal{A}_{f}\left(t_{0}+\Delta ; t_{0}, x_{0}\right) .
\end{aligned}
$$

Both the Value Function and the Attainable Set are, from the computational point of view, extremely complex objects, and, thus, approximation schemes are required. With respect to the latter, the literature offers several approaches, notably, polyhedral of either inner or outer type, [21], [22], ellipsoidal, [23], and "cloud of points" as endpoints of trajectory segments generated by constant controls. The last one was chosen as the one computationally more efficient for the AS-MPC scheme. For the former, methods for solving the Hamilton-JacobiBellman equation can be used. Albeit numerically complex, several methods, [24], [25], notably level set methods, are currently available in optimized software packages. In general, we may consider a number of value functions for given typified situations. In real-time "mission" execution, the relevant Value Function is identified via sensed data and invoked to compute the next optimal control at any $(t, x)$.

The AS-MPC scheme is follows:

1. Initialization: $t_{0}, x\left(t_{0}\right)$

2. Solve $\left(P_{T}^{\Delta}\right)$ over $\left[t_{0}, t_{0}+\Delta\right]$ to obtain $z^{*}$ and compute $u^{*}$ steering the system from $t_{0}$ to $t_{0}+\Delta$.

3. Apply $u^{*}$ during $\left[t_{0}, t_{0}+\Delta\right]$

4. Sample $x$ at $t_{0}+\Delta$ to obtain $\bar{x}=x\left(t_{0}+\Delta\right)$

5. Slide time, i.e., $t_{0}=t_{0}+\Delta$, estimate the new $x_{0}$, update the Attainable Set with the new $x_{0}$ (in some cases with simple translation and rotation), update the Value Function at the new $t_{0}+\Delta$, and goto 2 .

It is important to observe if $\left[0, T_{f}\right]$ is the mission time horizon, the AS-MPC achieves the true optimum in the absence of perturbations if $T_{f}$ is chosen instead of $t_{0}+T$ in the computation of the Value Function. It is clear that, in general, the realtime computational burden of this scheme is extremely low as it involves only very simple computational operations. For relatively small values of $\Delta$ the on-line operations of updating the Value Function and the Attainable Set are computationally inexpensive. In figure 2, it is shown: (i) the Forward Attainable
Set for the unicycle, and (ii) the Value Function in the absence of obstacles. The controls to be applied to the vehicle are

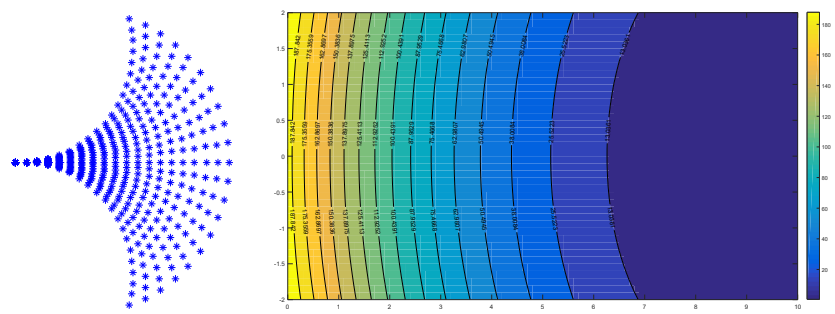

Fig. 2. AUV Forward Attainable Set, and Position control Value Function

found by searching for the minimum value within the vehicle's Forward Attainable Set.

Next we present the asymptotic optimality and stability properties of the presented AS-MPC scheme derived in [6]. In what follows, we consider the mission running on $\left[0, T_{f}\right)$ with $T_{f}=\infty$. Denote by $\left(x_{T, \Delta}^{*}, u_{T, \Delta}^{*}\right)$ the associated MPC optimal control process. Let $J(x, u)$ be the value of integral form equivalent of the cost functional associated with the $(x, u)$, by $J(x, u)_{\mid[\alpha, \beta]}$ its restriction to the interval $[\alpha, \beta]$, and by $J_{k}(x, u)$ its restriction to the interval $[k \Delta,(k+1) \Delta]$.

Proposition 1. Asymptotic Optimality. Let $\left(x^{*}, u^{*}\right)$ be an optimal control process such that $\lim _{t \rightarrow \infty} x^{*}(t)=\xi^{*}$, where $\xi^{*}$ is an equilibrium point in $C_{\infty}$. Then,

$$
\begin{aligned}
& \text { 1. } \lim _{\Delta \downarrow 0, T \uparrow \infty} \sum_{k=1}^{\infty} J_{k}\left(x_{T, \Delta}^{*}, u_{T, \Delta}^{*}\right)=J\left(x^{*}, u^{*}\right) \\
& \text { 2. } \lim _{k \rightarrow \infty}\left|J_{k}\left(x_{T, \Delta}^{*}, u_{T, \Delta}^{*}\right)-J\left(x^{*}, u^{*}\right)_{\mid[k \Delta,(k+1) \Delta]}\right|=0 \text {. }
\end{aligned}
$$

To facilitate the exposition, we consider $(g, f)$ to depend only $(x, u)$ and that either $g_{0}(\cdot) \equiv 0$ or $g_{0}$ is absorbed into the integral term. The stability property of the closed loop AS-MPC requires the following additional assumptions that denote be $(E S)$ :

- $(g, f)(\cdot, \cdot)$ is $C_{2}$ and $f$ is linearly controllable at $(0,0)$

- $\exists K_{g}$ such that $g(x, u) \leq K_{g}\left(\|x\|^{2}+\|u\|^{2}\right)$

- $\forall T>0, \forall x_{0}, \exists$ solution $\left(\bar{x}_{T}, \bar{u}_{T}\right)$ to $\left(P_{T}\right)$ with $\bar{x}(0)=x_{0}$, being $J_{T}\left(x_{0}\right)$ the cost.

Let $\mathcal{D}_{T}=\left\{x \in \boldsymbol{R}^{n}: J_{T}(x)<\infty\right\}, \mathcal{D}_{T, a}=\left\{x \in \mathbb{R}^{n}: J_{T}(x)<a\right\}$.

Proposition 2. Exponential Stability. Assume that (ES) holds and take some $a>0$. Then,

- $\forall \Delta>0, \exists \tilde{T}<\infty$ s.t., $\forall T \geq \tilde{T}$, the $(T, \Delta)-A S-M P C$ is exponentially stabilizable.

- Moreover, $\forall b$ s.t. $\mathcal{D}_{T-\Delta, b} \subset \mathcal{D}_{\infty, a}, \mathcal{D}_{T-\Delta, b} \subset$ Attraction Domain of $(T, \Delta)-A S-M P C$.

We remark, that slightly different variants of these two results st if we consider the appropriate time and state space discretizations. Since we are using the cloud of points as approximation to the Attainable Set, a good estimate of the Hausdorff distance between these sets to determine the worst case of sub-optimality.

Let $\Omega_{\varepsilon}$ denote the set $\left\{u_{i} \in \Omega: i=1, \ldots, N_{\varepsilon}\right\}$ satisfying the following properties: (i) $\Omega \subset \bigcup_{i=1}^{N_{\varepsilon}}\left(u_{i}+\varepsilon B\right.$ ), and (ii) 
$\forall i \exists j$ s.t. $\left\|f\left(x, u_{i}\right)-f\left(x, u_{j}\right)\right\|<\varepsilon$. Denote by $\mathcal{A}_{f}\left(t_{1} ; t_{0}, x\right)$ and $\mathcal{A}_{f}^{\varepsilon}\left(t_{1} ; t_{0}, x\right)$ the points attainable at $t_{1}>t_{0}$ from $x$ at $t_{0}$, by the dynamic system with controls, respectively, in $L^{\infty}$ with values in $\Omega$, and piecewise constant with values in $\Omega_{\varepsilon}$.

Proposition 3. Let $\Delta$ be a positive number. Under mild assumptions on the dynamics, we have, for any $(t, x) \in \boldsymbol{R} \times \boldsymbol{R}^{n}$,

$$
d_{H}\left(\mathcal{A}_{f}(t+\Delta ; t, x), \mathcal{A}_{f}^{\varepsilon}(t+\Delta ; t, x)\right) \leq \Delta \varepsilon e^{K_{f} \Delta} .
$$

Another key issue concerns the fact the point $\bar{x} \in \mathbb{R}^{n}$ to which the system is steered at a given time is very likely not listed in the stored Value Function look-up table. Thus, the following result on estimates of the space extrapolation of the Value Function is important.

Proposition 4. Assume that $V_{T}$ at $\bar{x}$ is not known, and that there is a grid of points $G_{\delta}$ in $\mathbb{R}^{n}$ such that the maximum distance between neighboring points in $G_{\delta}$ is less than $\delta>0$. Then, there is a simplex $S_{\bar{x}}=\left\{x_{i}: i=1, \ldots n+1\right\}$ which are the closest to $\bar{x}$ s.t. the estimate $\tilde{V}$ of $V$ at $\bar{x}$ is given by

$$
\tilde{V}(\bar{x})=\frac{\sum_{i=1}^{n+1} V_{i}\left\|\bar{x}-x_{i}\right\|^{-1}}{\sum_{i=1}^{n+1}\left\|\bar{x}-x_{i}\right\|^{-1}}
$$

where, for $i=1, \ldots, n+1, V_{i}=V\left(x_{i}\right)+\nabla V\left(x_{i}\right) \cdot \bar{v}_{i}$, with $\bar{v}_{i}=\bar{x}-x_{i}$ and the $n \times(n+1)$ unknowns of the vectors $\nabla V\left(x_{i}\right), i=1, \ldots, n+1$ are given as a solution of the set of $n+1$ set of equations $\nabla V\left(x_{i}\right) \cdot\left(\bar{v}_{i}-\bar{v}_{k}\right)=\frac{V\left(x_{k}\right)-V\left(x_{i}\right)}{\left\|x_{i}-x_{k}\right\|}$. Moreover, we have that, for some $c>0$,

$$
\|V(\bar{x})-\tilde{V}(\bar{x})\| \leq \max _{x_{i}, x_{j} \in S_{\bar{x}}}\left\{\left|V\left(x_{i}\right)-V\left(x_{j}\right)\right|\right\}+c \delta .
$$

\section{The Control Architecture}

The control architecture emerges from the application of the AS-MPC scheme to a hybrid automata model of the system which specifies the desired modes of operations and the discrete events triggering the transitions between them. It is worth remarking that the overall optimization incorporated in the minimum cost to go plays a role in determining controlled discrete events triggering the switch between modes in the present of unanticipated "external" events. Thus, the motion control of the generalized vehicle is organized into simpler AUV formation control problems. This amounts to regard the overall model of the formation as a hybrid dynamic control system, and a set of either controlled or uncontrolled associated discrete events. Thus, the implementation of the AS-MPC controller described in section III in the context of hybrid dynamics requires the need of an event-driven control strategy ensuring liveness and nonblocking properties, to be embedding of the AS-MPC controller.

To eliminate the burden inherent to the explanation of general contexts, we focus in the motion control of a three AUVs formation in a plane carrying the required navigation and payload sensors whose mission consists in gathering data along a given path such that the observation requirements are satisfied. We consider the following tasks: (i) Gather data along a given path. The AUVs keep the triangle formation and

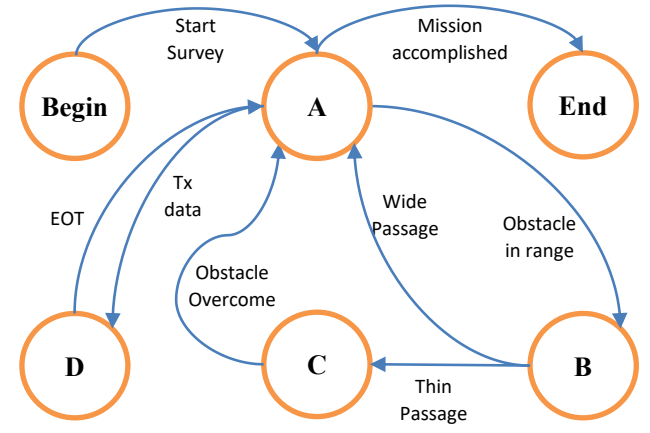

Fig. 3. Formation Pattern Automaton

the decentralized controller as described in section II ensures the simultaneous path tracking and formation maintenance; (ii) Avoid collision with obstacles. This task, involves obstacle detection, and characterization, path replanning, and, possibly, reconfiguration of the formation; (iii) Communicate with the external systems. This task is required to either transmit gathered data to enable the mission follow-up and to receive commands to change the mission if necessary.

The set of discrete modes and the events triggering the transition between them are represented in figure 3. Once the mission starts, the AUVs enters in the nominal mode $\mathbf{A}$ of data gathering while tracking the given path on a triangle formation. The Mission Accomplished event prompts the AUVs to the recovery operation. The follow-up of the mission requires the monitoring of the data being gathered. This is done in mode $\mathbf{D}$ and it means that, from time to time, one of the AUV surfaces, transmits the gathered data as well as the health status of the vehicles, which after a scrutiny, might entail a change in the mission. The occurrence instants can be pre-planned or the result of either controlled or uncontrolled events. Once the exchange of information is complete, the surfacing vehicle joins the other two AUVs that, in the meantime, had been waiting loitering, in order to pursue the operation mode $\mathbf{A}$. If an Obstacle in range event is detected by any of the AUVs, then the system moves to mode $\mathbf{B}$. In this mode, the obstacle is characterized and a collision avoidance path is computed. Then, two events might occur: either a Wide passage is available and the formation is kept unchanged and the systems moves to the nominal mode A tracking the original path, or a Thin passage is available and the system transits to mode $\mathbf{C}$ where the formation is reconfigured to overcome de obstacle. Once this action is completed the systems resumes its operation in the nominal mode $\mathbf{A}$.

Special attention is paid to the obstacle collision avoidance due to the fact that it illustrates well the point concerning the interaction between mission planning and control. We impose the following assumptions: (i) Data for obstacle detection and characterization is obtained by using a range finder; (ii) The unmapped obstacles in the environment are relatively sparse; (iii) Obstacles are locally modelled by circles; (iv) The range finder sensor reaches a distance larger than that transversed by the vehicle within the time interval of length $\Delta$. 


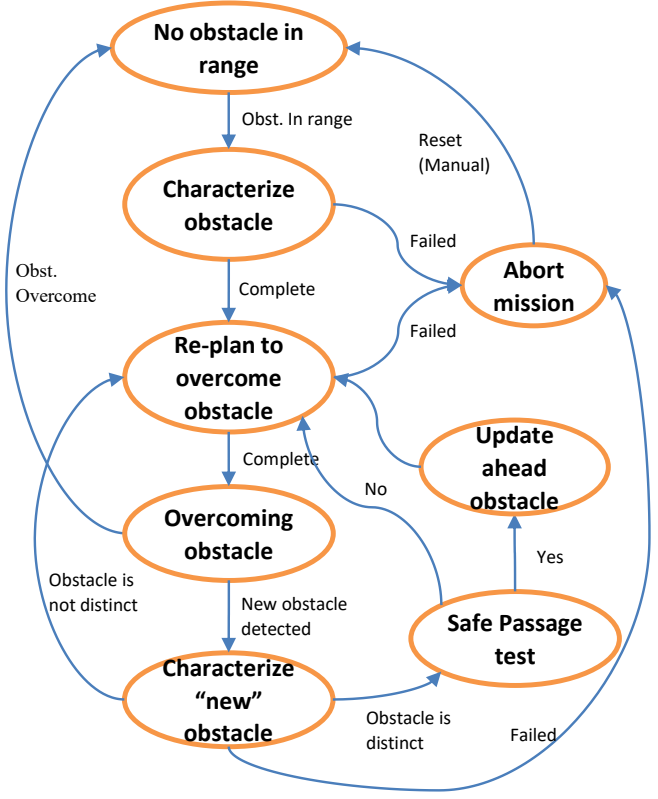

Fig. 4. Obstacle Collision Avoidance Automaton

The automaton diagram in figure 4 shows the various modes and associated transition events. Once an obstacle is detected, it is characterized to compute the best strategy to avoid collision and remain as close as possible to the path to be tracked. Remark that the formation pattern can be deformed to characterize the obstacle and to overcome it in an optimal way. These issues are essential to a better replanning of the path of each AUV. When an AUV is close to an obstacle, then its ASMPC is modified by adding penalization function guaranteeing a safety distance $d_{s}$ to the obstacle. This procedure easily handles multiple obstacles.

Simulation results obtained with the proposed control structure are shown in figure 5. The mission consists in gathering data while tracking a path defined by the line segment joining points $\mathrm{A}$ and $\mathrm{B}$ in a given triangle formation.

After deployment, the AUVs are loitering in the triangle formation for the survey around the departure point A. Once the survey starts at time $t_{1}$, mode $\mathbf{A}$ is activated and the triangle formation tracks the given path to the final destination B. At time $t_{2}$, the leading vehicle detects obstacle $O_{1}$. Then, the formation switches to mode $\mathbf{B}$ to characterize the obstacle. In order to do this efficiently the AUV formation changes to a transversal line formation and, once this is done, a path to overcome it is defined by the RAS-MPC by computing minimum of a mapping obtained by adding a penalization to the Value Function. Since there is plenty of space, at time $t_{3}$ the AUVs return to the triangle formation while circumventing the obstacle and trying to reach the mission path.

At time $t_{4}$, obstacle $\mathrm{O}_{2}$ is detected on the right. RASMPC determines that the best path is between $O_{1}$ and $O_{2}$. Moreover, the safety criteria above determines that the passage between $O_{1}$ and $O_{2}$ is safe for the whole formation, and the triangle formation is kept. At time $t_{4}^{\prime}$, a third obstacle $O_{3}$

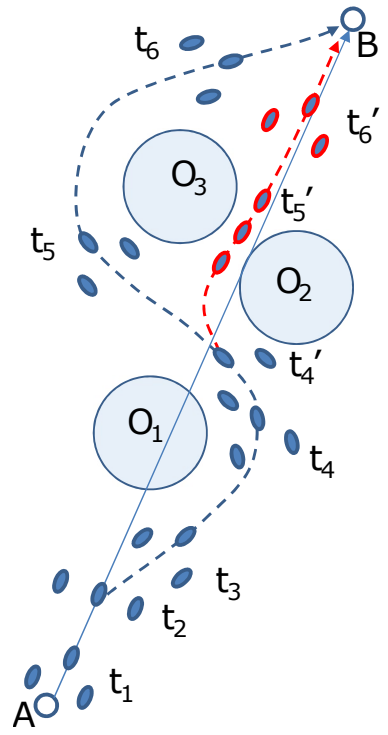

Fig. 5. Obstacle avoidance simulations results

is detected and its characterization in mode $\mathbf{B}$ together with the safety criteria determines two different scenarios to pursue the mission:(i) Follow through a thin passage between $\mathrm{O}_{2}$ and $\mathrm{O}_{3}$ safe for a single vehicle. Choosing it means changing to longitudinal line formation that results in being closer to the mission path but with loss of quality of the gathered data due to the adopted formation; (ii) Circumvent $\mathrm{O}_{3}$ by the left that would entail a longer route far away from the mission path, but would allow to preserve the triangle formation with a higher quality of the data gathered.

A simple onboard optimization procedure determines that the first option is the best one. Once the obstacles are overcome at time $t_{6}^{\prime}$, the formation resumes to the normal triangle until it reaches the final destination $\mathrm{B}$ where the mission mode changes to $\mathbf{D}$ to proceed with data transmission. In this state all the vehicles surface, transmit data, and remain loitering around the final destination $\mathrm{B}$.

Now, we present some simulation results for the AUV unicycle model showing the effect of noise in the AS-MPC behavior. The AUV is steered from an initial point to the final point in an optimal fashion in two situations: first, without state constraints and then, with state constraints. In the latter case, these were incorporated by considering a penalization term in the cost functional so that the optimization process prevents the state constraints violation. Here, the Value Function is computed by solving several instances of $\left(P_{T}\right)$ with multiple initial conditions in a $4 \mathrm{~m}$ by $10 \mathrm{~m}$ state space region.

In the first case - without state constraints - the following levels of additive Gaussian noise were considered:

- No noise: $\left(\mu_{x}, \sigma_{x}\right)=(0,0)$ and $\left(\mu_{y}, \sigma_{y}\right)=(0,0)$ - solid line trajectory.

- Noise level 1: $\left(\mu_{x}, \sigma_{x}\right)=(0,0.01)$ and $\left(\mu_{y}, \sigma_{y}\right)=(0,0.01)$ - dashed line trajectory.

- Noise level 2: $\left(\mu_{x}, \sigma_{x}\right)=(0,0.01)$ and $\left(\mu_{y}, \sigma_{y}\right)=$ $(-0.01,0.01)$ - dotted line trajectory. 
- Noise level 3: $\left(\mu_{x}, \sigma_{x}\right)=(0,0.01)$ and $\left(\mu_{y}, \sigma_{y}\right)=$ $(-0.02,0.01)$ - dash-dot line trajectory.

In figure 6 we can observe that the trajectory error increases as the noise level increases. Moreover, the effect of a non zero mean noise greatly impacts the tracking performance when compared with a standard deviation only noise.

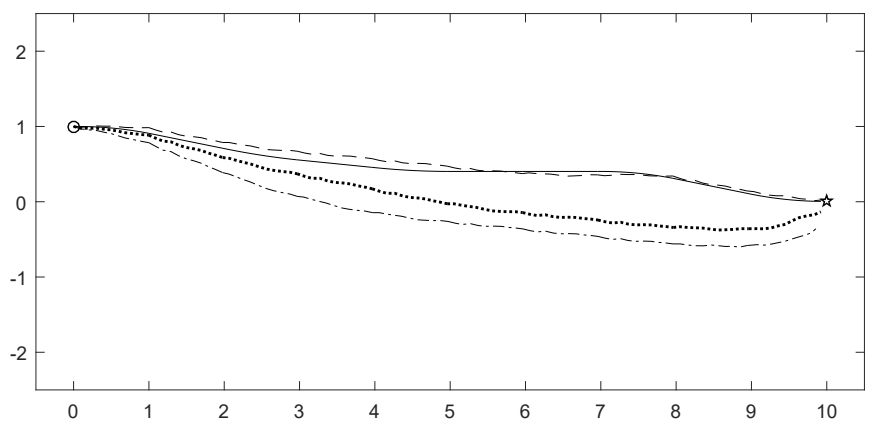

Fig. 6. Trajectory without obstacles: noiseless, noise levels 1, 2, 3

For the case of the space cluttered with obstacles, it is interesting to note that, in the absence of noise, the trajectory passes between obstacles and close to them, while the presence of noise forces it to pass away from the obstacles. The considered Gaussian noise level for this example is $\left(\mu_{x}, \sigma_{x}\right)=(0,0.01)$ and $\left(\mu_{y}, \sigma_{y}\right)=(-0.015,0.01)$ (sub-optimal).

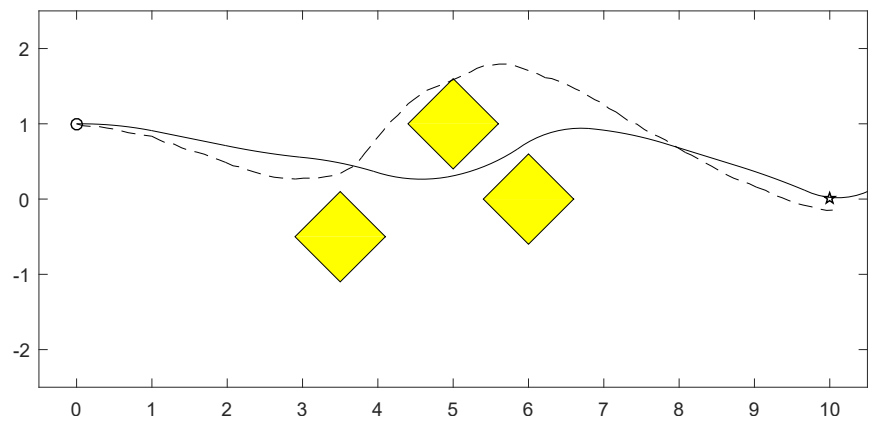

Fig. 7. Trajectory with obstacles: a) noiseless (solid), b) noise level (dashed)

\section{CONCLuSions}

The investigation of the AS-MPC scheme presented for path tracking control of a single AUV or formation of AUVs was further extended into two directions: Stability and the effect of perturbations. The key issue of the AS-MPC is the mitigation of the real-time computational burden and the ability of adapting to unmapped obstacle avoidance. While the former is motivated by limited onboard energy and computational power, in a context of strict real-time constraints, it shows the flexibility of the RAS-MPC scheme with a control architecture to handle unmapped obstacle as well as its robustness to additive noise. The obtained simulation results are encouraging and point to the next step: migrate the developments to a multiple AUV based system for field testing.

\section{REFERENCES}

[1] D. Mayne, J. Rawlings, C. Rao, and P. Scokaert, "Constrained model predictive control: Stability and optimality," Automatica, vol. 36, no. 6, pp. 789-814, 2000.

[2] E. Franco, L. Magni, T. Parisini, M. Polycarpou, and D. Raimondo, "Cooperative constrained control of distributed agents with nonlinear dynamics and delayed information exchange: A stabilizing recedinghorizon approach," IEEE TAC, vol. 53, pp. 324-338, 2008.

[3] L. Gruene, J. Pannek, and K. Worthmann, "A networked constrained nonlinear MPC scheme," in European Control Conf., Budapest, Hungary, 2009.

[4] R. Gomes and F. Pereira, "A hybrid systems model predictive control framework for auv motion control," in Procs ECC 2018, Limassol, Cyprus, June 12-15 2018.

[5] - "A Reach Set MPC Scheme For The Cooperative Control Of Autonomous Underwater Vehicles," in Procs PhysCon 2017, Florence, Italy, July 2017.

[6] R. Gomes, "An AUV systems model predictive control approach," Ph.D. dissertation, Faculty of Engineering, Porto University, 2018.

[7] T. Fossen, Guidance and Control of Ocean Vehicles. Wiley, 1994.

[8] R. Kristiansen and P. Nicklasson, "Spacecraft formation flying: A review and new results on state feedback control," Acta Astronautica, vol. 65, no. 11-12, pp. 1537 - 1552, 2009.

[9] W. Ren and R. Beard, "Virtual structure based spacecraft formation control with formation feedback," in AIAA Guidance, Navigation and Control Conf., Monterey CA, 2002, pp. 2002-4963.

[10] Y. Lv, Q. Hu, G. Ma, and J. Zhou, "6 dof synchronized control for spacecraft formation flying with input constraint and parameter uncertainties," ISA Trans., 2011.

[11] E. Franco, T. Parisini, and M. Polycarpou, "Cooperative control of discrete-time agents with delayed information exchange: A recedinghorizon approach," in IEEE CDC, 2004, pp. 4274-4279.

[12] T. Keviczky, F. Borrelli, and G. Balas, "Decentralized receding horizon control for large scale dynamically decoupled systems," Automatica, vol. 42, pp. 2105-2115, 2006.

[13] T. Keviczky, F. Borrelli, K. Fregene, D. Godbole, and G. Balas, "Decentralized receding horizon control and coordination of autonomous vehicle formations," IEEE Trans. Control Systems Tech., vol. 16, pp. 19-33, 2008.

[14] L. Consolini, F. Morbidi, D. Prattichizzo, and M. Tosques, "Leaderfollower formation control of nonholonomic mobile robots with input constraints," Automatica, vol. 44, no. 5, pp. 1343 - 1349, 2008.

[15] Z. Chao, L. Ming, Z. Shaolei, and Z. Wenguang, "Collision-free UAV formation flight control based on nonlinear MPC," in Conf. on Electronics, Communications and Control, Sept 2011, pp. 1951-1956.

[16] S. Quintero, D. Copp, and J. Hespanha, "Robust UAV coordination for target tracking using output-feedback model predictive control with moving horizon estimation," in ACC, 2015, pp. 3758-3764.

[17] S. Bertrand, J. Marzat, H. Piet-Lahanier, A. Kahn, and Y. Rochefort, "MPC strategies for cooperative guidance of autonomous vehicles," in AerospaceLab Journal, Issue 8, December 2014.

[18] R. Andrade, G. Raffo, and J. Rico, "Model predictive control of a tiltrotor uav for load transportation," in European Control Conf., June 2016, pp. 2165-2170.

[19] C. Shen, Y. Shi, and B. Buckham, "Path-following control of an AUV using multi-objective model predictive control," in 2016 American Control Conf. (ACC), July 2016, pp. 4507-4512.

[20] N. Krasovskii and A. Subbotin, Game-Theoretical Control Problems, ser. Springer Series in Soviet Mathematics. Springer-Verlag New York, 1988.

[21] V. Baturin, E. Goncharova, F. Pereira, and J. Sousa, "Polyhedral approximations to the reachable set of impulsive dynamic control systems," Autom. \& Remote Control, vol. 69, no. 3, 2006.

[22] T. Graettinger and B. Krogh, "Hyperplane method for reachable state estimation for linear time-invariant systems," J. of Optim. Theory and Appl., vol. 69, pp. 555-588, 1991.

[23] A. Kurzhanski and I. Vályi, Ellipsoidal Calculus for Estimation and Control. Birkhäuser, 1997.

[24] J. Sethian, Level Set Methods and Fast Marching Methods, 2nd ed. Cambridge University Press, 1999.

[25] I. Michel, A. Bayen, and C. Tomlin, "Computing reachable sets for continuous dynamics games using level sets methods," IEEE Trans. on Automatic Control, vol. 50, pp. 980-1001, 2005. 